\title{
Optimal Decision Model under Mixture Inventory System
}

\author{
P. K. Tripathy ${ }^{1}$, N. K. Sahoo ${ }^{2 *}$ \\ ${ }^{1}$ P.G. Department of Statistics, Utkal University, Bhubaneswar, India \\ ${ }^{2}$ Dep. of Mathematics, S.S.S. Mahavidyalaya, Dhenkanal, India \\ *Corresponding Author: narenmaths@yahoo.co.in
}

Available online at: www.isroset.org

Accepted 25/Aug/2018, Online 30/Aug/2018

\begin{abstract}
In this paper, the behavior of an inventory model in a production system with Weibull demand and time dependent deterioration is depicted. Holding cost is changed per unit time. Mathematical model of both crisp and fuzzy models have been developed to determine the optimal cycle time and optimal inventory cost. Fuzzy set theory is primarily concerned with imprecision and uncertainty. It provides the decision maker as a mixture inventory system which is used in modeling realworld problems as compared to the classical deterministic and probabilistic mathematical tools. The demand, deterioration rate, holding cost, unit cost and shortage cost are taken mixture and subsequently as pentagonal fuzzy numbers. Both graded mean integration and signed distance method are used to defuzzify the total cost function. Numerical illustrations are provided to illustrate the applications of the model with useful graphs and tables to validate the developed model. Sensitivity analysis is carried out to analyze the variability in the optimal solution with respect to change in various parameters.
\end{abstract}

Keywords- EOQ Model, Weibull Demand, Pentagonal Fuzzy Number, Graded Mean Integration, Signed Distance. AMS Classification No: 90B05

\section{INTRODUCTION}

Deterioration is a trending research area in inventory model. It is a challenging issue to develop inventory model for deteriorating items such as food stuffs, vegetables, food grains, medicines, fashion cosmetics and electronics items etc. Hariga [3] developed a model under general continuous time dependent demand and three replenishment policies. Jaggi, Aggarwal and Goel [5] studied replenishment model of deteriorating items with function of inflation induced demand. The model with three-parameter Weibull as well as parabolic deterioration and various demands and holding cost are proposed by Sahoo and Tripathy [15] \& [16].

One of the weaknesses of recent model which is vastly used in business world is the unrealistic assumption of the different parameters. Fuzzy logic can deal with information arising from cognition and computational perception, that is, imprecise, vague, uncertain, partially true, or without sharp boundaries. Fuzzy inventory models are more realistic than the conventional inventory models. The uncertainties are due to fuzziness, and such cases are expanded in the fuzzy set theory which was demonstrated by Zadeh [20] and Park [12]. Chang et al. [2] and Hsieh [4] developed model with fuzzy back order quantity and fuzzy production inventory. Kao and Hsu [7] proposed lot size reorder point inventory with fuzzy demands. Yao et al. [18] and Jaggi et al. [6] developed deteriorating model with some parameters as triangular fuzzy number. The analysis of deteriorating inventory model began with Yao and Lee [19] who developed fuzzy inventory with or without backorder with trapezoidal fuzzy number. Since then, many related research with various parameters was found in Behera et al. [1] Mohanty and Tripathy [9], Sahoo et al. [14] and Tripathy et al. [17]. Panda et al. [11] studied on Pentagonal fuzzy number and its corresponding matrices. Nagar and Surana [10] proposed fuzzy deteriorating inventory model with fluctuating demand and using inventory parameters as pentagonal fuzzy numbers, while, Mondal and Mandal [8] discussed properties and applications of pentagonal fuzzy number.

This model is discussed for items which deteriorate from the beginning of time cycle where demand and deterioration are considered as Weibull and time proportional respectively. Holding cost is also changed per unit of time. Further the demand, deterioration rate and all costs are taken as mixture and subsequently pentagonal fuzzy numbers. Both graded mean integration and signed distance method are used to defuzzify the total cost function. Mathematical model has been developed for determining the optimal cycle time and optimal total inventory fuzzy cost. Numerical illustrations 
are given to validate the developed model. The useful graphs and tables with sensitivity analysis are carried out to analyze the variability in the optimal solution with respect to change in various system parameters.

\section{PRELIMINARIES}

Definition 1: (By $\mathrm{Pu}$ and $\mathrm{Liu}^{13}$ ) A fuzzy set $\tilde{a}$ defined on real number set $R$ is called a fuzzy point if its membership function is

$$
\mu_{\tilde{a}}(x)=\left\{\begin{array}{l}
0, \text { if } x<a \\
1, \text { if } x=a \\
0, \text { if } x>a
\end{array}\right\}
$$

where the point $a$ is called the support of fuzzy set $\tilde{a}$.

Definition 2: A fuzzy set $\left[a_{1 \alpha}, a_{2 \alpha}\right]$ where $0 \leq \alpha \leq 1$ and $a_{1}<a_{2}$ defined on $R$ is called a level of a fuzzy interval if its membership function is

$$
\mu_{\left[a_{1 \alpha}, a_{2 \alpha}\right]}(x)=\left\{\begin{array}{l}
\alpha, a_{1} \leq x \leq a_{2} \\
0, \text { otherwise }
\end{array}\right\}
$$

Definition 3: A triangular fuzzy number $\widetilde{A}=\left(a_{1}, a_{2}, a_{3}\right)$, $a_{1}<a_{2}<a_{3}$ defined on $R$ with membership function $\mu_{\tilde{A}}$ as:

$$
\mu_{\tilde{A}}(x)=\left\{\begin{array}{l}
0, \text { if } x<a_{1} \\
\frac{x-a_{1}}{a_{2}-a_{1}}, \text { if } x \in\left[a_{1}, a_{2}\right] \\
\frac{a_{3}-x}{a_{3}-a_{2}}, \text { if } x \in\left[a_{2}, a_{3}\right] \\
0, \text { if } x>a_{3}
\end{array}\right.
$$

The $\alpha$-cut of $\tilde{A}=\left(a_{1}, a_{2}, a_{3}\right), 0 \leq \alpha \leq 1$ is

$$
A(\alpha)=\left[A_{L}(\alpha), A_{R}(\alpha)\right]
$$

where $A_{L}(\alpha)=a_{1}+\left(a_{2}-a_{1}\right) \alpha$

and $A_{R}(\alpha)=a_{3}-\left(a_{3}-a_{2}\right) \alpha$ are the left and right end points of $A(\alpha)$.

Definition 4: A trapezoidal fuzzy number is depicted by $\tilde{A}=\left(a_{1}, a_{2}, a_{3}, a_{4}\right) \quad, \quad a_{1}<a_{2}<a_{3}<a_{4} \quad$ with membership function $\mu_{\tilde{A}}$ is defined on $\mathrm{R}$ as:

$$
\mu_{\tilde{A}}(x)= \begin{cases}0, & \text { if } x<a_{1} \\ L(x)=\frac{x-a_{1}}{a_{2}-a_{1}}, & \text { if } x \in\left[a_{1}, a_{2}\right] \\ 1, & \text { if } x \in\left[a_{2}, a_{3}\right] \\ R(x)=\frac{a_{4}-x}{a_{4}-a_{3}}, & \text { if } x \in\left[a_{3}, a_{4}\right] \\ 0 \quad, & \text { if } x>a_{4}\end{cases}
$$

The $\alpha$-cut of $\tilde{A}=\left(a_{1}, a_{2}, a_{3}, a_{4}\right) 0<\alpha<1$ is

$$
A(\alpha)=\left[A_{L}(\alpha), A_{R}(\alpha)\right]
$$

where $A_{L}(\alpha)=a_{1}+\left(a_{2}-a_{1}\right) \alpha$

and $A_{R}(\alpha)=a_{4}-\left(a_{4}-a_{3}\right) \alpha$ are the left and right end points of $A(\alpha)$.

Definition 5: A pentagonal fuzzy number is depicted by $\widetilde{A}=\left(a_{1}, a_{2}, a_{3}, a_{4}, a_{5}\right) a_{1}<a_{2}<a_{3}<a_{4}<a_{5}$ is obtained on $\mathrm{R}$ with membership function $\mu_{\tilde{A}}$ as:

$$
\mu_{\tilde{A}}(x)=\left\{\begin{array}{l}
0, \text { if } x<a_{1} \\
L_{1}(x)=\frac{x-a_{1}}{a_{2}-a_{1}}, \quad \text { if } x \in\left[a_{1}, a_{2}\right] \\
L_{2}(x)=\frac{x-a_{2}}{a_{3}-a_{2}}, \quad \text { if } x \in\left[a_{2}, a_{3}\right] \\
1, \text { if } x=a_{3} \\
R_{1}(x)=\frac{a_{4}-x}{a_{4}-a_{3}}, \text { if } x \in\left[a_{3}, a_{4}\right] \\
R_{2}(x)=\frac{a_{5}-x}{a_{5}-a_{4}}, \text { if } x \in\left[a_{4}, a_{5}\right] \\
0 \quad, \text { if } x>a_{5}
\end{array}\right.
$$

The $\alpha$-cut of $\tilde{A}=\left(a_{1}, a_{2}, a_{3}, a_{4}, a_{5}\right)$, for $0<\alpha<1$ is $A(\alpha)=\left[A_{L}(\alpha), A_{R}(\alpha)\right]$, where $A_{L}(\alpha)=\frac{A_{L_{1}}(\alpha)+A_{L_{2}}(\alpha)}{2}$ and $A_{R}(\alpha)=\frac{A_{R_{1}}(\alpha)+A_{R 2}(\alpha)}{2}$ are the left and right end points of $A(\alpha)$ such that

$$
\begin{gathered}
A_{L_{1}}(\alpha)=a_{1}+\left(a_{2}-a_{1}\right) \alpha ; \\
A_{L_{2}}(\alpha)=a_{2}+\left(a_{3}-a_{2}\right) \alpha \\
\text { and } A_{R_{1}}(\alpha)=a_{4}-\left(a_{4}-a_{3}\right) \alpha ;
\end{gathered}
$$
$A_{R_{2}}(\alpha)=a_{5}-\left(a_{5}-a_{4}\right) \alpha$. Figure 1 portrays a pentagonal inventory during production cycle 


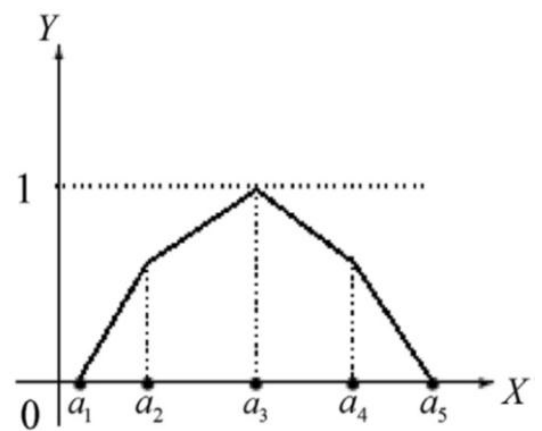

Figure 1. Pentagonal fuzzy number

Definition 6: If $\widetilde{A}=\left(a_{1}, a_{2}, a_{3}, a_{4}, a_{5}\right)$ be a pentagonal fuzzy number, then the graded mean integration method of $\widetilde{A}$ is defined as:

$$
G_{M}(\tilde{A})=\frac{\frac{1}{2} \int_{0}^{w_{A}} \alpha\left[A_{L}(\alpha)+A_{R}(\alpha)\right] d \alpha}{\int_{0}^{1} \alpha d \alpha}
$$

with $0<\alpha \leq w_{A}$ and $0<w_{A} \leq 1$

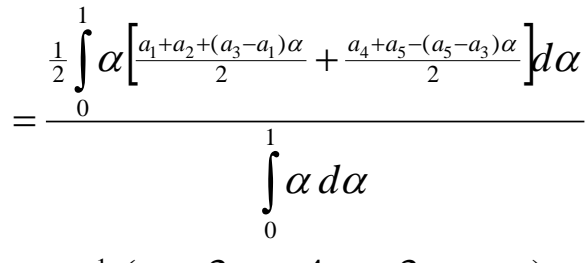

$$
=\frac{1}{12}\left(a_{1}+3 a_{2}+4 a_{3}+3 a_{4}+a_{5}\right)
$$

Definition 7: The signed distance method of $\tilde{A}$ with pentagonal fuzzy number $\tilde{A}=\left(a_{1}, a_{2}, a_{3}, a_{4}, a_{5}\right)$ is evaluated as:

$$
\begin{gathered}
S_{D}(\tilde{A})=\frac{1}{2} \int_{0}^{w_{A}}\left[A_{L}(\alpha)+A_{R}(\alpha)\right] d \alpha \\
\text { with } 0<\alpha \leq w_{A} \text { and } 0<w_{A} \leq 1 \\
=\frac{1}{2} \int_{0}^{1}\left[\frac{a_{1}+a_{2}+\left(a_{3}-a_{1}\right) \alpha}{2}+\frac{a_{4}+a_{5}-\left(a_{5}-a_{3}\right) \alpha}{2}\right] d \alpha \\
=\frac{1}{8}\left(a_{1}+2 a_{2}+2 a_{3}+2 a_{4}+a_{5}\right)
\end{gathered}
$$

\section{NOTATIONS AND ASSUMPTIONS}

\section{Notations}

(i)

$d(t)$ is the demand rate at any time.

(ii) $\theta$ is the deterioration rate per unit time.

(iii) $A, c, h$ and $S$ are the ordering cost, unit cost, holding cost and shortage cost respectively per unit time. (iv) $\quad t_{2}$ is the length of the order cycle.

(v) $Q$ is the ordering quantity per unit.

(vi) $\quad C\left(t_{1}, t_{2}\right)$ is the total inventory cost per unit time.

(vii) $\quad d$ is the fuzzy demand per unit time.

(viii) $\theta$ is the fuzzy deterioration rate per unit time.

(ix) $\quad \tilde{c}, \tilde{h}$ and $\tilde{S}$ are the fuzzy unit cost, fuzzy holding cost and fuzzy shortage cost respectively per unit time.

(x) $\quad C\left(t_{1}, t_{2}\right)$ is the total fuzzy inventory cost per unit time.

(xi) $\quad C_{G M}\left(t_{1}, t_{2}\right)$ is the defuzzified value of

$C\left(t_{1}, t_{2}\right)$ by applying graded mean integration method.

(xii) $C_{S D}\left(t_{1}, t_{2}\right)$ is the defuzzified value of $\tilde{C}\left(t_{1}, t_{2}\right)$ by applying signed distance method.

\section{Assumptions}

(xiii) Demand $\quad d(t)=\alpha \beta t^{\beta-1} \quad$ is $\quad$ Weibull distribution function where $\alpha>0$ is a scale parameter and $\beta>0$ is a shape parameter.

$\theta(t)=\theta t, 0<\theta<1$ is the deterioration rate per unit time.

(xv) $\quad h(t)=h t$ is the time proportional holding cost per unit time.

(xvi) The replenishment rate is instantaneous and lead-time is zero.

(xvii) Shortages are allowed and fully backlogged.

\section{MATHEMATICAL MODEL}

Let $I(t)$ be the inventory level at any time $t$ with initial inventory $Q$. The inventory level gradually diminishes during the period $\left[0, t_{1}\right]$ due to demand and deterioration and exhausted at time $t_{1}$. Finally, a shortage is occurred during the interval of time period $\left[t_{1}, t_{2}\right]$, which are fully backlogged. Since the inventory is depleted by the combined effect of demand and deterioration the inventory level $I(t)$ at time $t$ is governed by the following differential equations. Crisp Model

$\frac{d I(t)}{d t}+\theta I(t)=-d(t), 0 \leq t \leq t_{1}$

with $I(0)=Q$ and $I\left(t_{1}\right)=0$. 
And $\frac{d I(t)}{d t}=-d(t), t_{1} \leq t \leq t_{2}$

with $I\left(t_{1}\right)=0$

As $d(t)=\alpha \beta t^{\beta-1}$ and $\theta(t)=\theta t$ then the equations

and (2) becomes

$\frac{d I(t)}{d t}+\theta t I(t)=-\alpha \beta t^{\beta-1}, 0 \leq t \leq t_{1}$

with $I(0)=Q$ and $I\left(t_{1}\right)=0$.

And, $\frac{d I(t)}{d t}=-\alpha \beta t^{\beta-1}, t_{1} \leq t \leq t_{2}$

with $I\left(t_{1}\right)=0$.

Solution of equation (3) using condition $I(0)=Q$ is calculated as

$I(t)=Q e^{-\frac{\theta t^{2}}{2}}-\left(\alpha t^{\beta}+\frac{\alpha \beta \theta}{2(\beta+2)} t^{\beta+2}\right) e^{-\frac{\theta t^{2}}{2}}, 0 \leq t \leq t_{1}$

As $I\left(t_{1}\right)=0 \Rightarrow Q=\alpha t_{1}{ }^{\beta}+\frac{\alpha \beta \theta}{2(\beta+2)} t_{1}{ }^{\beta+2}$

Thus

$$
\begin{array}{r}
I(t)=\left[\left(\alpha t_{1}{ }^{\beta}+\frac{\alpha \beta \theta}{2(\beta+2)} t_{1}^{\beta+2}\right)-\left(\alpha t^{\beta}+\frac{\alpha \beta \theta}{2(\beta+2)} t^{\beta+2}\right)\right] e^{-\frac{\theta t^{2}}{2}} \\
=\left[\alpha\left(t_{1}{ }^{\beta}-t^{\beta}\right)+\frac{\alpha \beta \theta}{2(\beta+2)}\left(t_{1}^{\beta+2}-t^{\beta+2}\right)\right]\left(1-\frac{\theta t^{2}}{2}\right) \\
=\left[\begin{array}{l}
\alpha\left(t_{1}{ }^{\beta}-t^{\beta}\right)-\frac{\alpha \theta}{2}\left(t_{1}{ }^{\beta} t^{2}-t^{\beta+2}\right) \\
+\frac{\alpha \beta \theta}{2(\beta+2)}\left(t_{1}{ }^{\beta+2}-t^{\beta+2}\right)
\end{array}\right], 0 \leq t \leq t_{1}
\end{array}
$$

Solution of equation (4) will be

$$
I(t)=\alpha\left(t_{1}^{\beta}-t^{\beta}\right), t_{1} \leq t \leq t_{2}
$$

Inventory is available in the system during the time period $\left(0, t_{1}\right)$. The cost for holding in stock inventory is computed for time period $\left(0, t_{1}\right)$ only.

Total no. of holding cost unit $I_{H C}$ during period $\left[0, t_{2}\right]$ is given by

$$
\begin{aligned}
& I_{H C}=\int_{0}^{t_{1}} h(t) I(t) d t \\
& =h \int_{0}^{t_{1}} t\left[\begin{array}{l}
\left.\alpha\left(t_{1}{ }^{\beta}-t^{\beta}\right)-\frac{\alpha \theta}{2}\left(t_{1}{ }^{\beta} t^{2}-t^{\beta+2}\right)\right] d t \\
+\frac{\alpha \beta \theta}{2(\beta+2)}\left(t_{1}{ }^{\beta+2}-t^{\beta+2}\right)
\end{array}\right] \\
& =h\left[\frac{\alpha \beta}{2(\beta+2)} t_{1}^{\beta+2}+\frac{\alpha \beta \theta}{8(\beta+4)} t_{1}{ }^{\beta+4}\right]
\end{aligned}
$$

Total no. of deteriorated units $I_{D}$ during period $\left[0, t_{2}\right]$ is determined as

$$
\begin{gathered}
I_{D}=c[Q-\text { Total Demand }] \\
=c\left[Q-\int_{0}^{t_{1}} d(t) d t\right] \\
=\frac{\alpha \beta \varepsilon}{2(\beta+2)} t_{1}^{\beta+2}
\end{gathered}
$$

Total no. of units of shortage $I_{S}$ during period $\left[0, t_{2}\right]$ is illustrated as

$$
\begin{aligned}
I_{S} & =S \int_{t_{1}}^{t_{2}}-I(t) d t \\
& =-S\left(\alpha t_{1}{ }^{\beta} t_{2}-\frac{\alpha}{\beta+1} t_{2}^{\beta+1}-\frac{\alpha \beta}{\beta+1} t_{1}^{\beta+1}\right)
\end{aligned}
$$

Total cost of the system per unit time is calculated as

$$
\begin{gathered}
C\left(t_{1}, t_{2}\right)=\frac{1}{t_{2}}\left[A+I_{H C}+I_{D}+I_{S}\right] \\
\Rightarrow C\left(t_{1}, t_{2}\right)=\frac{1}{t_{2}}\left[\begin{array}{l}
A+\frac{\alpha \beta h}{2(\beta+2)} t_{1}^{\beta+2}+\frac{\alpha \beta \theta h}{8(\beta+4)} t_{1}^{\beta+4} \\
+\frac{\alpha \beta \theta c}{2(\beta+2)} t_{1}{ }^{\beta+2}-S\left(\begin{array}{l}
\alpha t_{1}{ }^{\beta} t_{2}-\frac{\alpha}{\beta+1} t_{2}{ }^{\beta+1} \\
-\frac{\alpha \beta}{\beta+1} t_{1}{ }^{\beta+1}
\end{array}\right)
\end{array}\right]
\end{gathered}
$$

\section{Fuzzy Model}

It is not easy to define all the system parameters precisely due to uncertainty in the environment. Accordingly it is assumed that some of these parameters namely $\tilde{\alpha}, \tilde{\beta}, \tilde{c}, \tilde{S}, \tilde{\theta}, \tilde{h}$ may change within some limits. Let $\tilde{\alpha}=\left(\alpha_{1}, \alpha_{2}, \alpha_{3}, \alpha_{4}, \alpha_{5}\right) \quad, \quad \tilde{\beta}=\left(\beta_{1}, \beta_{2}, \beta_{3}, \beta_{4}, \beta_{5}\right)$, $\tilde{h}=\left(h_{1}, h_{2}, h_{3}, h_{4}, h_{5}\right) \quad, \quad \tilde{c}=\left(c_{1}, c_{2}, c_{3}, c_{4}, c_{5}\right) \quad$, $\tilde{S}=\left(S_{1}, S_{2}, S_{3}, S_{4}, S_{5}\right) \quad, \quad \tilde{\theta}=\left(\theta_{1}, \theta_{2}, \theta_{3}, \theta_{4}, \theta_{5}\right) \quad$, are pentagonal fuzzy numbers.

Total cost of the system per unit time in fuzzy sense is governed by

$$
\tilde{C}\left(t_{1}, t_{2}\right)=\frac{1}{t_{2}}\left[\begin{array}{l}
A+\frac{\tilde{\alpha} \tilde{\beta} \tilde{h}}{2(\tilde{\beta}+2)} t_{1}^{\tilde{\beta}+2}+\frac{\tilde{\alpha} \tilde{\beta} \tilde{\theta} \tilde{h}}{8(\tilde{\beta}+4)} t_{1}^{\tilde{\beta}+4} \\
+\frac{\tilde{\alpha} \tilde{\beta} \tilde{\tilde{c}} \tilde{\beta}}{2(\tilde{\beta}+2)} t_{1}^{\tilde{\beta}+2}-\tilde{S}\left(\begin{array}{l}
\tilde{\alpha} t_{1}^{\tilde{\beta}} t_{2}-\frac{\tilde{\alpha}}{\tilde{\beta}+1} t_{2}^{\tilde{\beta}+1} \\
-\frac{\tilde{\alpha} \tilde{\beta}}{\tilde{\beta}+1} t_{1}^{\tilde{\beta}+1}
\end{array}\right)
\end{array}\right]
$$


The fuzzy total cost $\tilde{C}\left(t_{1}, t_{2}\right)$ is defuzzified by graded mean integration as well as signed distance method

By graded mean integration method, the total cost is given as

$$
\begin{aligned}
& C_{G M}\left(t_{1}, t_{2}\right)=\frac{1}{12}\left(C_{G M_{1}}\left(t_{1}, t_{2}\right)+3 C_{G M_{2}}\left(t_{1}, t_{2}\right)+4 K_{G M_{3}}\left(t_{1}, t_{2}\right)+3 C_{G M_{4}}\left(t_{1}, t_{2}\right)+K_{G M_{5}}\left(t_{1}, t_{2}\right)\right) \\
& =\frac{1}{12 t_{2}}\left[\begin{array}{l}
12 A+\left\{\frac{\alpha_{1} \beta_{1} h_{1}}{2\left(\beta_{1}+2\right)} t_{1}^{\beta_{1}+2}+\frac{\alpha_{1} \beta_{1} \theta_{1} h_{1}}{8\left(\beta_{1}+4\right)} t_{1}^{\beta_{1}+4}+\frac{\alpha_{1} \beta_{1} \theta_{1} c_{1}}{2\left(\beta_{1}+2\right)} t_{1}^{\beta_{1}+2}-S_{1}\left(\alpha_{1} t_{1}^{\beta_{1}} t_{2}-\frac{\alpha_{1}}{\beta_{1}+1} t_{2}^{\beta_{1}+1}-\frac{\alpha_{1} \beta_{1}}{\beta_{1}+1} t_{1}^{\beta_{1}+1}\right)\right\} \\
+3\left\{\frac{\alpha_{2} \beta_{2} h_{2}}{2\left(\beta_{2}+2\right)} t_{1}^{\beta_{2}+2}+\frac{\alpha_{2} \beta_{2} \theta_{2} h_{2}}{8\left(\beta_{2}+4\right)} t_{1}^{\beta_{2}+4}+\frac{\alpha_{2} \beta_{2} \theta_{2} c_{2}}{2\left(\beta_{2}+2\right)} t_{1}^{\beta_{2}+2}-S_{2}\left(\alpha_{2} t_{1}^{\beta_{2}} t_{2}-\frac{\alpha_{2}}{\beta_{2}+1} t_{2}^{\beta_{2}+1}-\frac{\alpha_{2} \beta_{2}}{\beta_{2}+1} t_{1}^{\beta_{2}+1}\right)\right\} \\
+4\left\{\frac{\alpha_{3} \beta_{3} h_{3}}{2\left(\beta_{3}+2\right)} t_{1}^{\beta_{3}+2}+\frac{\alpha_{3} \beta_{3} \theta_{3} h_{3}}{8\left(\beta_{3}+4\right)} t_{1}^{\beta_{3}+4}+\frac{\alpha_{3} \beta_{3} \theta_{3} c_{3}}{2\left(\beta_{3}+2\right)} t_{1}^{\beta_{3}+2}-S_{3}\left(\alpha_{3} t_{1}^{\beta_{3}} t_{2}-\frac{\alpha_{3}}{\beta_{3}+1} t_{2}^{\beta_{3}+1}-\frac{\alpha_{3} \beta_{3}}{\beta_{3}+1} t_{1}^{\beta_{3}+1}\right)\right\} \\
+3\left\{\frac{\alpha_{4} \beta_{4} h_{4}}{2\left(\beta_{4}+2\right)} t_{1}^{\beta_{4}+2}+\frac{\alpha_{4} \beta_{4} \theta_{4} h_{4}}{8\left(\beta_{4}+4\right)} t_{1}^{\beta_{4}+4}+\frac{\alpha_{4} \beta_{4} \theta_{4} c_{4}}{2\left(\beta_{4}+2\right)} t_{1}^{\beta_{4}+2}-S_{4}\left(\alpha_{4} t_{1}^{\beta_{4}} t_{2}-\frac{\alpha_{4}}{\beta_{4}+1} t_{2}^{\beta_{4}+1}-\frac{\alpha_{4} \beta_{4}}{\beta_{4}+1} t_{1}^{\beta_{4}+1}\right)\right\} \\
+\left\{\frac{\alpha_{5} \beta_{5} h_{5}}{2\left(\beta_{5}+2\right)} t_{1}^{\beta_{5}+2}+\frac{\alpha_{5} \beta_{5} \theta_{5} h_{5}}{8\left(\beta_{5}+4\right)} t_{1}^{\beta_{5}+4}+\frac{\alpha_{5} \beta_{5} \theta_{5} c_{5}}{2\left(\beta_{5}+2\right)} t_{1}^{\beta_{5}+2}-S_{5}\left(\alpha_{5} t_{1}^{\beta_{5}} t_{2}-\frac{\alpha_{5}}{\beta_{5}+1} t_{2}^{\beta_{5}+1}-\frac{\alpha_{5} \beta_{5}}{\beta_{5}+1} t_{1}^{\beta_{5}+1}\right)\right\}
\end{array}\right]
\end{aligned}
$$

The optimal values of $t_{1}$ and $t_{2}$ can be obtained so as to minimize the total fuzzy cost $C_{G M}\left(t_{1}, t_{2}\right)$ by solving the following equations

$$
\frac{\partial C_{G M}\left(t_{1}, t_{2}\right)}{\partial t_{1}}=0 \text { and } \frac{\partial C_{G M}\left(t_{1}, t_{2}\right)}{\partial t_{2}}=0
$$

Equation (15) becomes

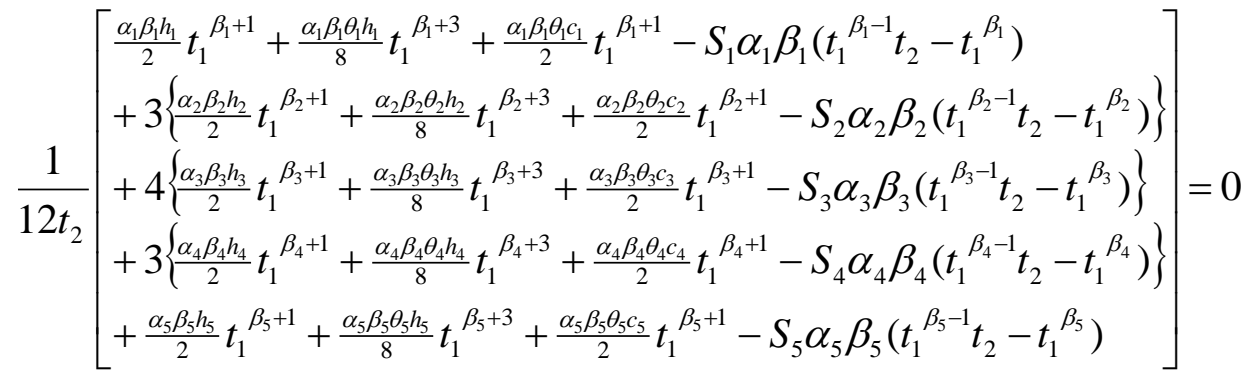

$$
\begin{aligned}
& \text { \& } \\
& \frac{1}{12 t_{2}}\left[\begin{array}{l}
S_{1} \alpha_{1}\left(t_{1}^{\beta_{1}}-t_{2}^{\beta_{1}}\right)+3 S_{2} \alpha_{2}\left(t_{1}^{\beta_{2}}-t_{2}^{\beta_{2}}\right)+4 S_{3} \alpha_{3}\left(t_{1}^{\beta_{3}}-t_{2}^{\beta_{3}}\right) \\
+3 S_{4} \alpha_{4}\left(t_{1}^{\beta_{4}}-t_{2}^{\beta_{4}}\right)+S_{5} \alpha_{5}\left(t_{1}^{\beta_{5}}-t_{2}{ }^{\beta_{5}}\right)
\end{array}\right] \\
& +\frac{1}{12 t_{2}^{2}}\left[\begin{array}{l}
12 A+\left\{\frac{\alpha_{1} \beta_{1} h_{1}}{2\left(\beta_{1}+2\right)} t_{1}^{\beta_{1}+2}+\frac{\alpha_{1} \beta_{1} \theta_{1} h_{1}}{8\left(\beta_{1}+4\right)} t_{1}^{\beta_{1}+4}+\frac{\alpha_{1} \beta_{1} \theta_{1} c_{1}}{2\left(\beta_{1}+2\right)} t_{1}^{\beta_{1}+2}-S_{1}\left(\alpha_{1} t_{1}^{\beta_{1}} t_{2}-\frac{\alpha_{1}}{\beta_{1}+1} t_{2}^{\beta_{1}+1}-\frac{\alpha_{1} \beta_{1}}{\beta_{1}+1} t_{1}^{\beta_{1}+1}\right)\right\} \\
+3\left\{\frac{\alpha_{2} \beta_{2} h_{2}}{2\left(\beta_{2}+2\right)} t_{1}^{\beta_{2}+2}+\frac{\alpha_{2} \beta_{2} \theta_{2} h_{2}}{8\left(\beta_{2}+4\right)} t_{1}^{\beta_{2}+4}+\frac{\alpha_{2} \beta_{2} \theta_{2} c_{2}}{2\left(\beta_{2}+2\right)} t_{1}^{\beta_{2}+2}-S_{2}\left(\alpha_{2} t_{1}^{\beta_{2}} t_{2}-\frac{\alpha_{2}}{\beta_{2}+1} t_{2}^{\beta_{2}+1}-\frac{\alpha_{2} \beta_{2}}{\beta_{2}+1} t_{1}^{\beta_{2}+1}\right)\right\} \\
+4\left\{\frac{\alpha_{3} \beta_{3} h_{3}}{2\left(\beta_{3}+2\right)} t_{1}^{\beta_{3}+2}+\frac{\alpha_{3} \beta_{3} \theta_{3} h_{3}}{8\left(\beta_{3}+4\right)} t_{1}^{\beta_{3}+4}+\frac{\alpha_{3} \beta_{3} \theta_{3} c_{3}}{2\left(\beta_{3}+2\right)} t_{1}^{\beta_{3}+2}-S_{3}\left(\alpha_{3} t_{1}^{\beta_{3}} t_{2}-\frac{\alpha_{3}}{\beta_{3}+1} t_{2}^{\beta_{3}+1}-\frac{\alpha_{3} \beta_{3}}{\beta_{3}+1} t_{1}^{\beta_{3}+1}\right)\right\} \\
+3\left\{\frac{\alpha_{4} \beta_{4} h_{4}}{2\left(\beta_{4}+2\right)} t_{1}^{\beta_{4}+2}+\frac{\alpha_{4} \beta_{4} \theta_{4} h_{4}}{8\left(\beta_{4}+4\right)} t_{1}^{\beta_{4}+4}+\frac{\alpha_{4} \beta_{4} \theta_{4} c_{4}}{2\left(\beta_{4}+2\right)} t_{1}^{\beta_{4}+2}-S_{4}\left(\alpha_{4} t_{1}^{\beta_{4}} t_{2}-\frac{\alpha_{4}}{\beta_{4}+1} t_{2}^{\beta_{4}+1}-\frac{\alpha_{4} \beta_{4}}{\beta_{4}+1} t_{1}^{\beta_{4}+1}\right)\right\} \\
+\left\{\frac{\alpha_{5} \beta_{5} h_{5}}{2\left(\beta_{5}+2\right)} t_{1}^{\beta_{5}+2}+\frac{\alpha_{5} \beta_{5} \theta_{5} h_{5}}{8\left(\beta_{5}+4\right)} t_{1}^{\beta_{5}+4}+\frac{\alpha_{5} \beta_{5} \theta_{5} c_{5}}{2\left(\beta_{5}+2\right)} t_{1}^{\beta_{5}+2}-S_{5}\left(\alpha_{5} t_{1}^{\beta_{5}} t_{2}-\frac{\alpha_{5}}{\beta_{5}+1} t_{2}^{\beta_{5}+1}-\frac{\alpha_{5} \beta_{5}}{\beta_{5}+1} t_{1}^{\beta_{5}+1}\right)\right\}
\end{array}\right]=0
\end{aligned}
$$

Further, for the convexity of total fuzzy cost function $C_{G M}\left(t_{1}, t_{2}\right)$, the following conditions must be satisfied 


$$
\begin{gathered}
\frac{\partial^{2} C_{G M}\left(t_{1}, t_{2}\right)}{\partial t_{1}^{2}}>0, \frac{\partial^{2} C_{G M}\left(t_{1}, t_{2}\right)}{\partial t_{2}{ }^{2}}>0 \\
\left(\frac{\partial^{2} C_{G M}\left(t_{1}, t_{2}\right)}{\partial t_{1}{ }^{2}}\right)\left(\frac{\partial^{2} C_{G M}\left(t_{1}, t_{2}\right)}{\partial t_{2}{ }^{2}}\right)-\left(\frac{\partial^{2} C_{G M}\left(t_{1}, t_{2}\right)}{\partial t_{1} \partial t_{2}}\right)>0
\end{gathered}
$$

It is difficult to prove the convexity mathematically, since it is complicated to determine the second derivatives of the total fuzzy cost function $C_{G M}\left(t_{1}, t_{2}\right)$. It has constrained to show the convexity of total fuzzy cost in graph (Figure 2).

(ii) By signed distance method, the total fuzzy cost is illustrated as

$$
\begin{aligned}
C_{S D}\left(t_{1}, t_{2}\right)=\frac{1}{8}\left(C_{G M_{1}}\left(t_{1}, t_{2}\right)+2 C_{G M_{2}}\left(t_{1}, t_{2}\right)+2 K_{G M_{3}}\left(t_{1}, t_{2}\right)+2 C_{G M_{4}}\left(t_{1}, t_{2}\right)+K_{G M_{5}}\left(t_{1}, t_{2}\right)\right) \\
\left.\begin{array}{l}
8 A+\left\{\frac{\alpha_{1} \beta_{1} h_{1}}{2\left(\beta_{1}+2\right)} t_{1}^{\beta_{1}+2}+\frac{\alpha_{1} \beta_{1} \theta_{1} h_{1}}{8\left(\beta_{1}+4\right)} t_{1}^{\beta_{1}+4}+\frac{\alpha_{1} \beta_{1} \theta_{1} c_{1}}{2\left(\beta_{1}+2\right)} t_{1}^{\beta_{1}+2}-S_{1}\left(\alpha_{1} t_{1}^{\beta_{1}} t_{2}-\frac{\alpha_{1}}{\beta_{1}+1} t_{2}^{\beta_{1}+1}-\frac{\alpha_{1} \beta_{1}}{\beta_{1}+1} t_{1}^{\beta_{1}+1}\right)\right\} \\
+2\left\{\frac{\alpha_{2} \beta_{2} h_{2}}{2\left(\beta_{2}+2\right)} t_{1}^{\beta_{2}+2}+\frac{\alpha_{2} \beta_{2} \theta_{2} h_{2}}{8\left(\beta_{2}+4\right)} t_{1}^{\beta_{2}+4}+\frac{\alpha_{2} \beta_{2} \theta_{2} c_{2}}{2\left(\beta_{2}+2\right)} t_{1}^{\beta_{2}+2}-S_{2}\left(\alpha_{2} t_{1}^{\beta_{2}} t_{2}-\frac{\alpha_{2}}{\beta_{2}+1} t_{2}^{\beta_{2}+1}-\frac{\alpha_{2} \beta_{2}}{\beta_{2}+1} t_{1}^{\beta_{2}+1}\right)\right\} \\
=\frac{1}{8 t_{2}}+ \\
+2\left\{\frac{\alpha_{3} \beta_{3} h_{3}}{2\left(\beta_{3}+2\right)} t_{1}^{\beta_{3}+2}+\frac{\alpha_{3} \beta_{3} \theta_{3} h_{3}}{8\left(\beta_{3}+4\right)} t_{1}^{\beta_{3}+4}+\frac{\alpha_{3} \beta_{3} \theta_{3} c_{3}}{2\left(\beta_{3}+2\right)} t_{1}^{\beta_{3}+2}-S_{3}\left(\alpha_{3} t_{1}^{\beta_{3}} t_{2}-\frac{\alpha_{3}}{\beta_{3}+1} t_{2}^{\beta_{3}+1}-\frac{\alpha_{3} \beta_{3}}{\beta_{3}+1} t_{1}^{\beta_{3}+1}\right)\right\} \\
+2\left\{\frac{\alpha_{4} \beta_{4} h_{4}}{2\left(\beta_{4}+2\right)} t_{1}^{\beta_{4}+2}+\frac{\alpha_{4} \beta_{4} \theta_{4} h_{4}}{8\left(\beta_{4}+4\right)} t_{1}^{\beta_{4}+4}+\frac{\alpha_{4} \beta_{4} \theta_{4} c_{4}}{2\left(\beta_{4}+2\right)} t_{1}^{\beta_{4}+2}-S_{4}\left(\alpha_{4} t_{1}^{\beta_{4}} t_{2}-\frac{\alpha_{4}}{\beta_{4}+1} t_{2}^{\beta_{4}+1}-\frac{\alpha_{4} \beta_{4}}{\beta_{4}+1} t_{1}^{\beta_{4}+1}\right)\right\} \\
+\left\{\frac{\alpha_{5} \beta_{5} h_{5}}{2\left(\beta_{5}+2\right)} t_{1}^{\beta_{5}+2}+\frac{\alpha_{5} \beta_{5} \theta_{5} h_{5}}{8\left(\beta_{5}+4\right)} t_{1}^{\beta_{5}+4}+\frac{\alpha_{5} \beta_{5} \theta_{5} c_{5}}{2\left(\beta_{5}+2\right)} t_{1}^{\beta_{5}+2}-S_{5}\left(\alpha_{5} t_{1}^{\beta_{5}} t_{2}-\frac{\alpha_{5}}{\beta_{5}+1} t_{2}^{\beta_{5}+1}-\frac{\alpha_{5} \beta_{5}}{\beta_{5}+1} t_{1}^{\beta_{5}+1}\right)\right\}
\end{array}\right]
\end{aligned}
$$

The optimal values of $t_{1}$ and $t_{2}$ can be obtained by solving the following equations to minimize the total fuzzy cost $C_{S D}\left(t_{1}, t_{2}\right)$.

$$
\frac{\partial C_{S D}\left(t_{1}, t_{2}\right)}{\partial t_{1}}=0 \text { and } \frac{\partial C_{S D}\left(t_{1}, t_{2}\right)}{\partial t_{2}}=0
$$

Equation (6.21) becomes

$$
\begin{aligned}
& \frac{1}{8 t_{2}}\left[\begin{array}{l}
\frac{\alpha_{1} \beta_{1} h_{1}}{2} t_{1}^{\beta_{1}+1}+\frac{\alpha_{1} \beta_{1} \theta_{1} h_{1}}{8} t_{1}^{\beta_{1}+3}+\frac{\alpha_{1} \beta_{1} \theta_{1} c_{1}}{2} t_{1}^{\beta_{1}+1}-S_{1} \alpha_{1} \beta_{1}\left(t_{1}^{\beta_{1}-1} t_{2}-t_{1}^{\beta_{1}}\right) \\
+2\left\{\frac{\alpha_{2} \beta_{2} h_{2}}{2} t_{1}^{\beta_{2}+1}+\frac{\alpha_{2} \beta_{2} \theta_{2} h_{2}}{8} t_{1}^{\beta_{2}+3}+\frac{\alpha_{2} \beta_{2} \theta_{2} c_{2}}{2} t_{1}^{\beta_{2}+1}-S_{2} \alpha_{2} \beta_{2}\left(t_{1}^{\beta_{2}-1} t_{2}-t_{1}^{\beta_{2}}\right)\right\} \\
+2\left\{\frac{\alpha_{3} \beta_{3} h_{3}}{2} t_{1}^{\beta_{3}+1}+\frac{\alpha_{3} \beta_{3} \theta_{3} h_{3}}{8} t_{1}^{\beta_{3}+3}+\frac{\alpha_{3} \beta_{3} \theta_{3} c_{3}}{2} t_{1}^{\beta_{3}+1}-S_{3} \alpha_{3} \beta_{3}\left(t_{1}^{\beta_{3}-1} t_{2}-t_{1}^{\beta_{3}}\right)\right\} \\
+2\left\{\frac{\alpha_{4} \beta_{4} h_{4}}{2} t_{1}^{\beta_{4}+1}+\frac{\alpha_{4} \beta_{4} \theta_{4} h_{4}}{8} t_{1}^{\beta_{4}+3}+\frac{\alpha_{4} \beta_{4} \theta_{4} c_{4}}{2} t_{1}^{\beta_{4}+1}-S_{4} \alpha_{4} \beta_{4}\left(t_{1}^{\beta_{4}-1} t_{2}-t_{1}^{\beta_{4}}\right)\right\} \\
+\frac{\alpha_{5} \beta_{5} h_{5}}{2} t_{1}^{\beta_{5}+1}+\frac{\alpha_{5} \beta_{5} \theta_{5} h_{5}}{8} t_{1}^{\beta_{5}+3}+\frac{\alpha_{5} \beta_{5} \theta_{5} c_{5}}{2} t_{1}^{\beta_{5}+1}-S_{5} \alpha_{5} \beta_{5}\left(t_{1}^{\beta_{5}-1} t_{2}-t_{1}^{\beta_{5}}\right)
\end{array}\right]=0 \\
& \text { \& } \\
& \frac{1}{8 t_{2}}\left[\begin{array}{l}
S_{1} \alpha_{1}\left(t_{1}^{\beta_{1}}-t_{2}^{\beta_{1}}\right)+2 S_{2} \alpha_{2}\left(t_{1}^{\beta_{2}}-t_{2}^{\beta_{2}}\right)+2 S_{3} \alpha_{3}\left(t_{1}^{\beta_{3}}-t_{2}^{\beta_{3}}\right) \\
+2 S_{4} \alpha_{4}\left(t_{1}^{\beta_{4}}-t_{2}^{\beta_{4}}\right)+S_{5} \alpha_{5}\left(t_{1}^{\beta_{5}}-t_{2}^{\beta_{5}}\right)
\end{array}\right]
\end{aligned}
$$




$$
\left.+\frac{1}{8 t_{2}^{2}}+\begin{array}{l}
8 A+\left\{\frac{\alpha_{1} \beta_{1} h_{1}}{2\left(\beta_{1}+2\right)} t_{1}^{\beta_{1}+2}+\frac{\alpha_{1} \beta_{1} \theta_{1} h_{1}}{8\left(\beta_{1}+4\right)} t_{1}^{\beta_{1}+4}+\frac{\alpha_{1} \beta_{1} \theta_{1} c_{1}}{2\left(\beta_{1}+2\right)} t_{1}^{\beta_{1}+2}-S_{1}\left(\alpha_{1} t_{1}^{\beta_{1}} t_{2}-\frac{\alpha_{1}}{\beta_{1}+1} t_{2}^{\beta_{1}+1}-\frac{\alpha_{1} \beta_{1}}{\beta_{1}+1} t_{1}^{\beta_{1}+1}\right)\right\} \\
+2\left\{\frac{\alpha_{2} \beta_{2} h_{2}}{2\left(\beta_{2}+2\right)} t_{1}^{\beta_{2}+2}+\frac{\alpha_{2} \beta_{2} \theta_{2} h_{2}}{8\left(\beta_{2}+4\right)} t_{1}^{\beta_{2}+4}+\frac{\alpha_{2} \beta_{2} \theta_{2} c_{2}}{2\left(\beta_{2}+2\right)} t_{1}^{\beta_{2}+2}-S_{2}\left(\alpha_{2} t_{1}^{\beta_{2}} t_{2}-\frac{\alpha_{2}}{\beta_{2}+1} t_{2}^{\beta_{2}+1}-\frac{\alpha_{2} \beta_{2}}{\beta_{2}+1} t_{1}^{\beta_{2}+1}\right)\right\} \\
+2\left\{\frac{\alpha_{3} \beta_{3} h_{3}}{2\left(\beta_{3}+2\right)} t_{1}^{\beta_{3}+2}+\frac{\alpha_{3} \beta_{3} \theta_{3} h_{3}}{8\left(\beta_{3}+4\right)} t_{1}^{\beta_{3}+4}+\frac{\alpha_{3} \beta_{3} \theta_{3} c_{3}}{2\left(\beta_{3}+2\right)} t_{1}^{\beta_{3}+2}-S_{3}\left(\alpha_{3} t_{1}^{\beta_{3}} t_{2}-\frac{\alpha_{3}}{\beta_{3}+1} t_{2}^{\beta_{3}+1}-\frac{\alpha_{3} \beta_{3}}{\beta_{3}+1} t_{1}^{\beta_{3}+1}\right)\right\} \\
+2\left\{\frac{\alpha_{4} \beta_{4} h_{4}}{2\left(\beta_{4}+2\right)} t_{1}^{\beta_{4}+2}+\frac{\alpha_{4} \beta_{4} \theta_{4} h_{4}}{8\left(\beta_{4}+4\right)} t_{1}^{\beta_{4}+4}+\frac{\alpha_{4} \beta_{4} \theta_{4} c_{4}}{2\left(\beta_{4}+2\right)} t_{1}^{\beta_{4}+2}-S_{4}\left(\alpha_{4} t_{1}^{\beta_{4}} t_{2}-\frac{\alpha_{4}}{\beta_{4}+1} t_{2}^{\beta_{4}+1}-\frac{\alpha_{4} \beta_{4}}{\beta_{4}+1} t_{1}^{\beta_{4}+1}\right)\right\} \\
+\left\{\frac{\alpha_{5} \beta_{5} h_{5}}{2\left(\beta_{5}+2\right)} t_{1}^{\beta_{5}+2}+\frac{\alpha_{5} \beta_{5} \theta_{5} h_{5}}{8\left(\beta_{5}+4\right)} t_{1}^{\beta_{5}+4}+\frac{\alpha_{5} \beta_{5} \theta_{5} c_{5}}{2\left(\beta_{5}+2\right)} t_{1}^{\beta_{5}+2}-S_{5}\left(\alpha_{5} t_{1}^{\beta_{5}} t_{2}-\frac{\alpha_{5}}{\beta_{5}+1} t_{2}^{\beta_{5}+1}-\frac{\alpha_{5} \beta_{5}}{\beta_{5}+1} t_{1}^{\beta_{5}+1}\right)\right\}
\end{array}\right]
$$

Further, for the convexity of the total fuzzy cost function $C_{S D}\left(t_{1}, t_{2}\right)$, the following conditions must be satisfied

$$
\begin{aligned}
& \frac{\partial^{2} C_{S D}\left(t_{1}, t_{2}\right)}{\partial t_{1}{ }^{2}}>0, \frac{\partial^{2} C_{S D}\left(t_{1}, t_{2}\right)}{\partial t_{2}{ }^{2}}>0 \\
& \left(\frac{\partial^{2} C_{S D}\left(t_{1}, t_{2}\right)}{\partial t_{1}{ }^{2}}\right)\left(\frac{\partial^{2} C_{S D}\left(t_{1}, t_{2}\right)}{\partial t_{2}{ }^{2}}\right)-\left(\frac{\partial^{2} C_{S D}\left(t_{1}, t_{2}\right)}{\partial t_{1} \partial t_{2}}\right)>0
\end{aligned}
$$

It is difficult to prove the convexity mathematically as it is complicated to determine the second derivatives of the total fuzzy cost function $C_{S D}\left(t_{1}, t_{2}\right)$. Hence, the convexity of the total fuzzy cost is shown graphically (Figure 3 ).

\section{NUMERICAL ILLUSTRATIONS}

\section{Crisp Model:}

Illustration-1: To illustrate the solution process, setting $A=100, \alpha=50, \beta=0.25, \theta=0.25, C=10$, $h=2.5$ and $S=7.5$ with proper units and using Mathematica-5.1, the optimal replenishment time is determined as $t_{1}=1.29018, t_{2}=1.8739$ and minimum total cost is found as $C\left(t_{1}, t_{2}\right)=78.5062$.

Illustration-2: To understand the effect of solution process, assuming $A=200, \alpha=100, \beta=0.5$, $\theta=0.5, C=20, h=5$ and $S=15$ with proper units and using Mathematica-5.1, the optimal cycle time is evaluated as $t_{1}=0.807497, t_{2}=1.14238$. and optimum total cost is calculated as $C\left(t_{1}, t_{2}\right)=308.77$.

Illustration-3: Based on the computational process, considering $A=300, \alpha=150, \beta=0.75, \theta=0.75$, $C=30, h=7.5$ and $S=22.5$ with proper units and using Mathematica-5.1, the time period is determined as $t_{1}=0.623543, t_{2}=0.887471$ and cost value is found as $C\left(t_{1}, t_{2}\right)=663.909$.
Fuzzy Model:

By Graded Mean Integration Method (GMIM):

Illustration-4: To illustrate the solution process, setting $A=100 \quad, \quad \tilde{\alpha}=(30,40,50,60,70)$

$\tilde{\beta}=\left(\begin{array}{l}0.05,0.15,0.25, \\ 0.35,0.45\end{array}\right), \tilde{\theta}=\left(\begin{array}{l}0.05,0.15,0.25, \\ 0.35,0.45\end{array}\right)$

$, \tilde{C}=\left(\begin{array}{c}5,7.5,10, \\ 12.5,15\end{array}\right), \quad \tilde{h}=\left(\begin{array}{l}0.5,1.5,2.5, \\ 3.5,4.5\end{array}\right) \quad$ and

$\widetilde{S}=(5.5,6.5,7.5,8.5,9.5)$ with proper units and using Mathematica-5.1, the optimal replenishment time is obtained as $t_{1}=1.53738, t_{2}=2.65927$ and minimum fuzzy cost calculated as $C_{G M}\left(t_{1}, t_{2}\right)=76.9063$.

Illustration-5: To understand the effect of solution process, assuming $A=200, \tilde{\alpha}=\left(\begin{array}{l}60,80,100, \\ 120,120\end{array}\right)$, $\tilde{\beta}=\left(\begin{array}{l}0.1,0.3,0.5, \\ 0.7,0.9\end{array}\right) \quad, \quad \tilde{\theta}=\left(\begin{array}{l}0.1,0.3,0.5, \\ 0.7,0.9\end{array}\right)$, $\widetilde{C}=(10,15,20,25,30) \quad, \quad \tilde{h}=(1,3,5,7,9) \quad$ and $\tilde{S}=(11,13,15,17,19)$ with proper units and using Mathematica-5.1, the optimal cycle time is found as 
$t_{1}=0.725419, t_{2}=1.07932$ and optimal fuzzy cost is determined as $C_{G M}\left(t_{1}, t_{2}\right)=327.657$.

Illustration-6: Based on the computational process, considering $A=300 \quad, \quad \tilde{\alpha}=\left(\begin{array}{l}90,120,150, \\ 180,210\end{array}\right)$, $\tilde{\beta}=\left(\begin{array}{l}0.15,0.45,0.75, \\ 1.05,1.35\end{array}\right), \tilde{\theta}=\left(\begin{array}{l}0.15,0.45,0.75, \\ 1.05,1.35\end{array}\right)$ $, \tilde{C}=\left(\begin{array}{l}15,22.5,30, \\ 37.5,45\end{array}\right), \quad \tilde{h}=\left(\begin{array}{l}1.5,4.5,7.5 \\ 10.5,13.5\end{array}\right)$ and $\widetilde{S}=(16.5,19.5,22.5,25.5,28.5)$ with proper units and using Mathematica-5.1, the time period is obtained as $t_{1}=0.505067, t_{2}=0.724772$ and cost is calculated as $C_{G M}\left(t_{1}, t_{2}\right)=674.746$.

\section{By Signed Distance Method (SDM):}

Illustration-7: To illustrate the solution process, setting $A=100 \quad, \quad \tilde{\alpha}=(30,40,50,60,70)$ $\tilde{\beta}=\left(\begin{array}{l}0.05,0.15,0.25, \\ 0.35,0.45\end{array}\right), \tilde{\theta}=\left(\begin{array}{l}0.05,0.15,0.25, \\ 0.35,0.45\end{array}\right)$ $, \tilde{C}=\left(\begin{array}{l}5,7.5,10, \\ 12.5,15\end{array}\right), \quad \tilde{h}=\left(\begin{array}{l}0.5,1.5,2.5, \\ 3.5,4.5\end{array}\right)$ and $\tilde{S}=(5.5,6.5,7.5,8.5,9.5)$ with proper units and using Mathematica-5.1, the optimal replenishment time is evaluated as $t_{1}=1.35926, t_{2}=2.26523$ and minimum total fuzzy cost is determined as $C_{S D}\left(t_{1}, t_{2}\right)=80.5096$.

Illustration-8: To understand the effect of solution process, assuming $A=200, \tilde{\alpha}=\left(\begin{array}{l}60,80,100, \\ 120,120\end{array}\right)$, $\widetilde{\beta}=\left(\begin{array}{l}0.1,0.3,0.5, \\ 0.7,0.9\end{array}\right) \quad, \quad \tilde{\theta}=\left(\begin{array}{l}0.1,0.3,0.5, \\ 0.7,0.9\end{array}\right)$ $\widetilde{C}=(10,15,20,25,30) \quad, \quad \tilde{h}=(1,3,5,7,9) \quad$ and $\tilde{S}=(11,13,15,17,19)$ with proper units and using Mathematica-5.1, the optimum time is determined as $t_{1}=0.659012, t_{2}=0.965084$ and optimum fuzzy cost is found as $C_{S D}\left(t_{1}, t_{2}\right)=337.583$.

Illustration-9: Based on the computational process, considering $A=300 \quad, \quad \tilde{\alpha}=\left(\begin{array}{l}90,120,150, \\ 180,210\end{array}\right)$, $\tilde{\beta}=\left(\begin{array}{l}0.15,0.45,0.75, \\ 1.05,1.35\end{array}\right), \tilde{\theta}=\left(\begin{array}{l}0.15,0.45,0.75, \\ 1.05,1.35\end{array}\right)$
$, \tilde{C}=\left(\begin{array}{l}15,22.5,30, \\ 37.5,45\end{array}\right), \tilde{h}=\left(\begin{array}{l}1.5,4.5,7.5, \\ 10.5,13.5\end{array}\right)$ and $\widetilde{S}=(16.5,19.5,22.5,25.5,28.5)$ with proper units and using Mathematica-5.1, the time period is found as $t_{1}=0.505067, t_{2}=0.724772$ and total fuzzy cost is evaluated as $C_{S D}\left(t_{1}, t_{2}\right)=686.035$. Taking various parameters as pentagonal fuzzy numbers, the performance of two methods can be compared with optimal time period and optimal cost

Table 1: The optimum time period and optimum cost when $\tilde{\alpha}, \widetilde{\beta}, \tilde{\theta}, \tilde{h}, \tilde{c}, \tilde{S}$ are pentagonal fuzzy numbers

\begin{tabular}{|l|c|c|l|}
\hline $\begin{array}{l}\text { Observed } \\
\text { Method }\end{array}$ & $t_{1}$ & $t_{2}$ & $\begin{array}{l}\text { Optimal } \\
\text { Fuzzy cost }\end{array}$ \\
\hline GMIM & 0.725419 & 1.07932 & 327.657 \\
\hline SDM & 0.659012 & 0.965084 & 337.583 \\
\hline
\end{tabular}

Table 2: The optimum time period and optimum cost when $\tilde{\alpha}, \widetilde{\beta}, \tilde{\theta}, \tilde{h}, \tilde{c}$ are pentagonal fuzzy numbers

\begin{tabular}{|l|c|c|l|}
\hline $\begin{array}{l}\text { Observed } \\
\text { Method }\end{array}$ & $t_{1}$ & $t_{2}$ & $\begin{array}{l}\text { Optimal } \\
\text { Fuzzy cost }\end{array}$ \\
\hline GMIM & 0.723845 & 1.10348 & 323.284 \\
\hline SDM & 0.650257 & 0.976168 & 332.456 \\
\hline
\end{tabular}

Table 3: The optimum time period and optimum cost when $\tilde{\alpha}, \widetilde{\beta}, \tilde{\theta}, \tilde{h}$ are pentagonal fuzzy numbers

\begin{tabular}{|l|c|c|l|}
\hline $\begin{array}{l}\text { Observed } \\
\text { Method }\end{array}$ & $t_{1}$ & $t_{2}$ & $\begin{array}{l}\text { Optimal } \\
\text { Fuzzy cost }\end{array}$ \\
\hline GMIM & 0.763865 & 1.13657 & 312.609 \\
\hline SDM & 0.693558 & 1.01294 & 319.566 \\
\hline
\end{tabular}

Table 4: The optimum time period and optimum cost when $\tilde{\alpha}, \tilde{\beta}, \tilde{\theta}$ are pentagonal fuzzy numbers

\begin{tabular}{|l|c|c|l|}
\hline $\begin{array}{l}\text { Observed } \\
\text { Method }\end{array}$ & $t_{1}$ & $t_{2}$ & $\begin{array}{l}\text { Optimal } \\
\text { Fuzzy cost }\end{array}$ \\
\hline GMIM & 0.787699 & 1.15731 & 307.268 \\
\hline SDM & 0.718747 & 1.03489 & 313.084 \\
\hline
\end{tabular}

Table 5: The optimum time period and optimum cost when $\tilde{\alpha}, \widetilde{\beta}$ are pentagonal fuzzy numbers

\begin{tabular}{|l|c|c|l|}
\hline $\begin{array}{l}\text { Observed } \\
\text { Method }\end{array}$ & $t_{1}$ & $t_{2}$ & $\begin{array}{l}\text { Optimal } \\
\text { Fuzzy cost }\end{array}$ \\
\hline GMIM & 0.839459 & 1.20215 & 295.997 \\
\hline SDM & 0.776242 & 1.08508 & 299.037 \\
\hline
\end{tabular}




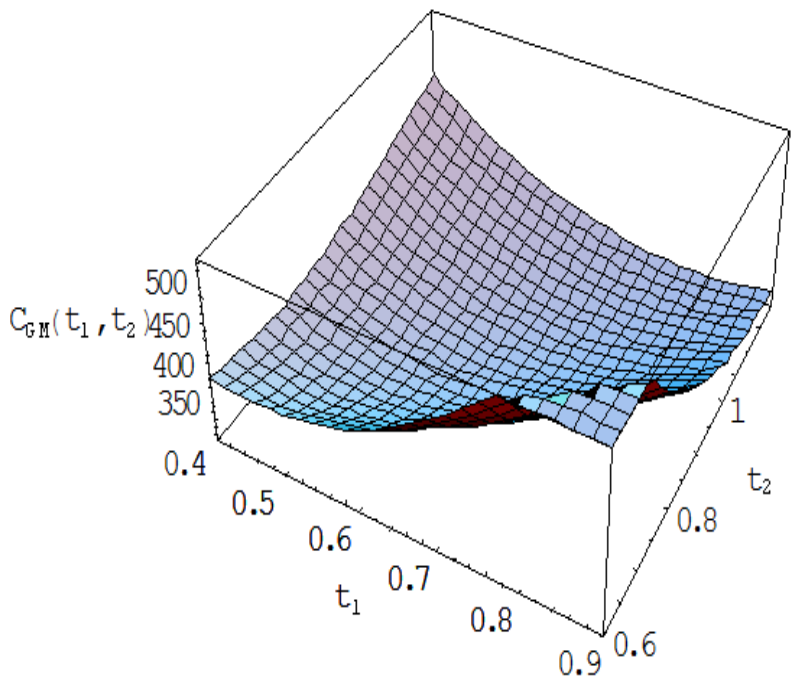

Figure 2. Total average $\cos t C_{G M}\left(t_{1}, t_{2}\right)$ vs. $t_{1}$ and $t_{2}$

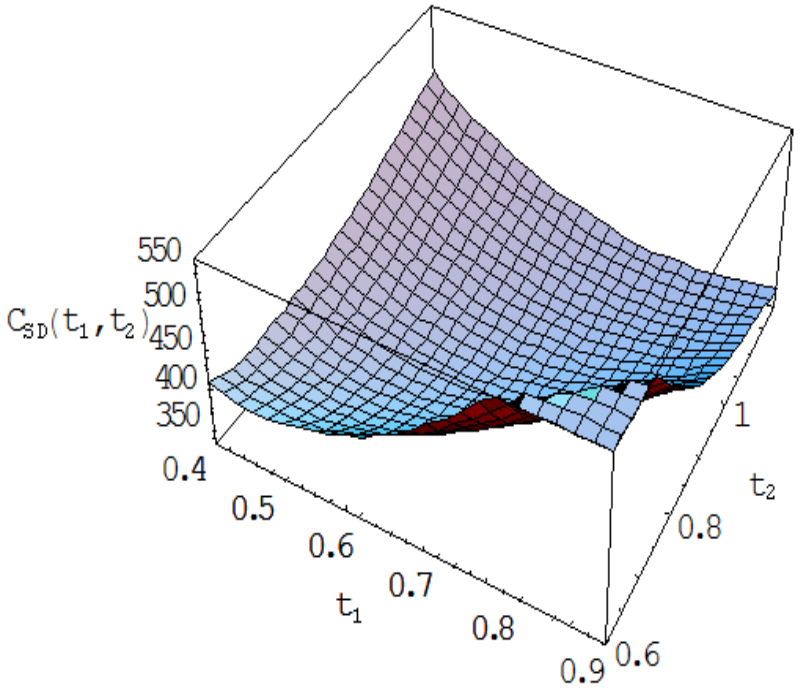

Figure 3. Total average $\cos t C_{S D}\left(t_{1}, t_{2}\right)$ vs. $t_{1}$ and $t_{2}$

VI. SENSITIVITY ANALYSIS

Table 6. (Sensitivity analysis by graded mean integration method)

\begin{tabular}{|c|c|c|c|c|c|}
\hline Parameters & $\%$ change in parameters & $t_{1}^{*}$ & $t_{2}^{*}$ & $C^{*}\left(t_{1}, t_{2}\right)$ & \% change in $C^{*}\left(t_{1}, t_{2}\right)$ \\
\hline \multirow{4}{*}{$A=200$} & +50 & 0.830305 & 1.30126 & 411.648 & +25.63382 \\
\hline & +25 & 0.781606 & 1.19592 & 371.605 & +13.41281 \\
\hline & -25 & 0.658268 & 0.946732 & 278.308 & -15.0612 \\
\hline & -50 & 0.573077 & 0.788821 & 220.713 & -32.639 \\
\hline \multirow{4}{*}{$\alpha=\left(\begin{array}{l}60,80,100 \\
120,140\end{array}\right)$} & +50 & 0.632417 & 0.897612 & 390.353 & +19.13464 \\
\hline & +25 & 0.672831 & 0.97487 & 360.895 & +10.14414 \\
\hline & -25 & 0.798552 & 1.23212 & 289 & -11.798 \\
\hline & -50 & 0.91248 & 1.48828 & 241.668 & -26.2436 \\
\hline \multirow{4}{*}{$\left(\begin{array}{l}0.1,0.3,0.5, \\
0.7,0.9\end{array}\right)$} & +50 & 0.637383 & 0.903344 & 358.561 & +9.431814 \\
\hline & +25 & 0.672094 & 0.971667 & 346.691 & +5.809124 \\
\hline & -25 & 0.770198 & 1.1595 & 298.876 & -8.78388 \\
\hline & -50 & 0.975775 & 1.64098 & 250.088 & -23.6738 \\
\hline \multirow{2}{*}{$\left(\begin{array}{l}0.1,0.3,0.5, \\
0.7,0.9\end{array}\right)$} & +50 & 0.641688 & 1.01273 & 354.689 & +8.250091 \\
\hline & +25 & 0.679193 & 1.04235 & 342.086 & +4.40369 \\
\hline
\end{tabular}




\begin{tabular}{|c|c|c|c|c|c|}
\hline \multirow{4}{*}{$h=(1,3,5,7,9)$} & -25 & 0.784668 & 1.12742 & 310.76 & -5.15692 \\
\cline { 2 - 6 } & -50 & 0.865169 & 1.19399 & 290.313 & -11.3973 \\
\cline { 2 - 6 } & +50 & 0.685016 & 1.04685 & 340.093 & +3.795432 \\
\cline { 2 - 6 } & +25 & 0.704186 & 1.0622 & 334.073 & +1.958145 \\
\hline \multirow{3}{*}{$c=\left(\begin{array}{l}10,15,20, \\
25,30\end{array}\right)$} & -25 & 0.749159 & 1.09862 & 320.786 & -2.09701 \\
\cline { 2 - 6 } & -50 & 0.77601 & 1.12065 & 313.383 & -4.35638 \\
\cline { 2 - 6 } & +50 & 0.643902 & 1.01491 & 354.308 & +8.133811 \\
\cline { 2 - 6 } & +25 & 0.68069 & 1.04382 & 341.845 & +4.330138 \\
\hline \multirow{3}{*}{$S=\left(\begin{array}{l}11,13,15, \\
17,19\end{array}\right)$} & -50 & 0.781464 & 1.12429 & 311.195 & -5.02416 \\
\cline { 2 - 6 } & +50 & 0.854565 & 1.18365 & 291.596 & -11.0057 \\
\cline { 2 - 6 } & +25 & 0.741458 & 0.988534 & 347.766 & +6.137211 \\
\cline { 2 - 6 } & -25 & 0.711461 & 1.16439 & 310.788 & +3.543034 \\
\hline
\end{tabular}

Table 7 (Sensitivity analysis by signed distance method)

\begin{tabular}{|c|c|c|c|c|c|}
\hline Parameters & $\%$ change in parameters & $t_{1}^{*}$ & $t_{2}^{*}$ & $C^{*}\left(t_{1}, t_{2}\right)$ & \% change in $C^{*}\left(t_{1}, t_{2}\right)$ \\
\hline \multirow{4}{*}{$A=200$} & +50 & 0.754444 & 1.16167 & 424.914 & +25.86949 \\
\hline & +25 & 0.710118 & 1.0684 & 383.255 & +13.52912 \\
\hline & -25 & 0.59798 & 0.847489 & 286.365 & -15.172 \\
\hline & -50 & 0.520609 & 0.707241 & 226.694 & -32.8479 \\
\hline \multirow{4}{*}{$\alpha=\left(\begin{array}{l}60,80,100 \\
120,140\end{array}\right)$} & +50 & 0.574495 & 0.803887 & 401.443 & +18.91683 \\
\hline & +25 & 0.611212 & 0.872456 & 371.451 & +10.0325 \\
\hline & -25 & 0.725539 & 1.10046 & 298.149 & -11.6813 \\
\hline & -50 & 0.829314 & 1.32712 & 249.796 & -26.0046 \\
\hline \multirow{4}{*}{$\beta=\left(\begin{array}{l}0.1,0.3,0.5 \\
0.7,0.9\end{array}\right)$} & +50 & 0.591209 & 0.830664 & 365.744 & +8.341948 \\
\hline & +25 & 0.617686 & 0.882715 & 355.256 & +5.235157 \\
\hline & -25 & 0.727595 & 1.10662 & 308.671 & -8.56441 \\
\hline & -50 & 0.855209 & 1.38921 & 261.432 & -22.5577 \\
\hline
\end{tabular}




\begin{tabular}{|c|c|c|c|c|c|}
\hline \multirow{4}{*}{$\theta=\left(\begin{array}{l}0.1,0.3,0.5, \\
0.7,0.9\end{array}\right)$} & +50 & 0.580899 & 0.900222 & 365.179 & +8.174582 \\
\hline & +25 & 0.615841 & 0.929105 & 352.328 & +4.367815 \\
\hline & -25 & 0.714501 & 1.01179 & 320.269 & -5.12881 \\
\hline & -50 & 0.790143 & 1.07627 & 299.245 & -11.3566 \\
\hline \multirow{4}{*}{$h=(1,3,5,7,9)$} & +50 & 0.622644 & 0.984716 & 349.318 & +3.476182 \\
\hline & +25 & 0.639931 & 0.949118 & 343.919 & +1.876872 \\
\hline & -25 & 0.680252 & 0.982943 & 330.808 & -2.00691 \\
\hline & -50 & 0.70414 & 1.00314 & 323.522 & -4.1652 \\
\hline \multirow{4}{*}{$c=\left(\begin{array}{l}10,15,20, \\
25,30\end{array}\right)$} & +50 & 0.582507 & 0.901713 & 364.821 & +8.068534 \\
\hline & +25 & 0.616935 & 0.930121 & 352.1 & +4.300276 \\
\hline & -25 & 0.712116 & 1.00957 & 320.683 & -5.00618 \\
\hline & -50 & 0.782127 & 1.06877 & 300.474 & -10.9926 \\
\hline \multirow{4}{*}{$S=\left(\begin{array}{l}11,13,15 \\
17,19\end{array}\right)$} & +50 & 0.675797 & 0.890949 & 358.303 & +6.13775 \\
\hline & +25 & 0.668744 & 0.92128 & 349.539 & +3.541648 \\
\hline & -25 & 0.644644 & 1.03424 & 320.229 & -5.14066 \\
\hline & -50 & 0.620993 & 1.1612 & 292.49 & -13.3576 \\
\hline
\end{tabular}

\section{RESULTS AND DISCUSSION}

The effects of changes in the system of parameters $A, \alpha, \beta, \theta, h, c$ and $S$ on the fuzzy costs derived by the proposed methods are now studied. The sensitivity analysis is performed by keeping all but one system parameters fixed at a time and study the change in the identified variable by fluctuating it from $25 \%$ to $50 \%$. The analysis is based on the results which are shown in Table -5.6 and Table-5.7.The sensitivity analysis for some parameters is presented in Table (5.6 \& 5.7) and some interesting findings are summarized as follows

(i) $t_{1}{ }^{*}, t_{2}{ }^{*}$ and fuzzy costs increase (decrease) drastically with the increase (decrease) with respect to the parameter $A$.

(ii) $t_{1}{ }^{*}$ and $t_{2}{ }^{*}$ drop down (move up) gradually while fuzzy costs move up (drop down) normally with the increase (decrease) of parameter $\alpha$. (iii) $t_{1}{ }^{*}$ and $t_{2}{ }^{*}$ decelerate (accelerate) regularly while fuzzy costs accelerate (decelerate) slightly with the increase (decrease) of parameter $\beta$.

(iv) $t_{1}{ }^{*}$ and $t_{2}{ }^{*}$ descend (ascend) slightly while fuzzy costs ascend (descend) gradually with the increase (decrease) of parameter $\theta$.

(v) $t_{1}{ }^{*}, t_{2}{ }^{*}$ decelerate (accelerate) and fuzzy costs accelerate (decelerate) moderately with the increases (decreases) of parameter $h$.

(vi) $t_{1}{ }^{*}$ and $t_{2}{ }^{*}$ drop down (move up) slowly while fuzzy costs move up (drop down) gradually with the increase (decrease) of parameter $c$.

(vii) $t_{1}{ }^{*}$ rises (falls) insensitively and $t_{2}{ }^{*}$ falls (rises) gradually while fuzzy costs accelerate (decelerate) strictly with the increase (decrease) with respect to parameter $S$. 
Among all the parameters, fuzzy total cost is especially sensitive to the variation of demand rate, deterioration rate, ordering cost, unit cost, holding cost, shortage cost and total fuzzy cost in both methods are represented by the following figures

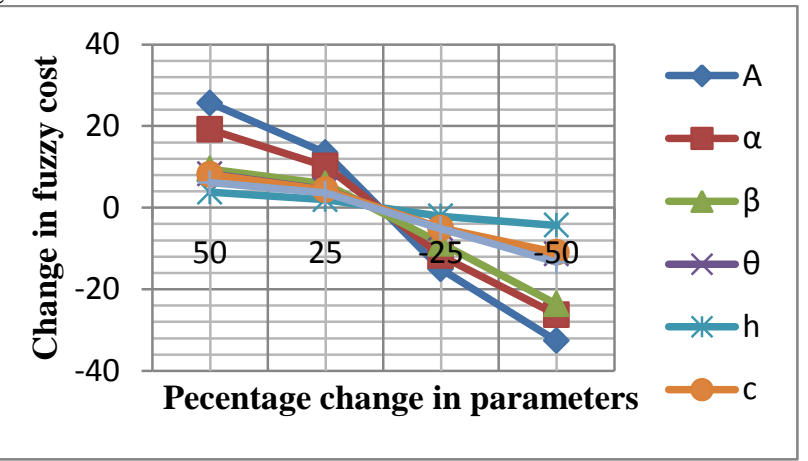

Figure 3. Behavior of fuzzy cost in graded mean integration method

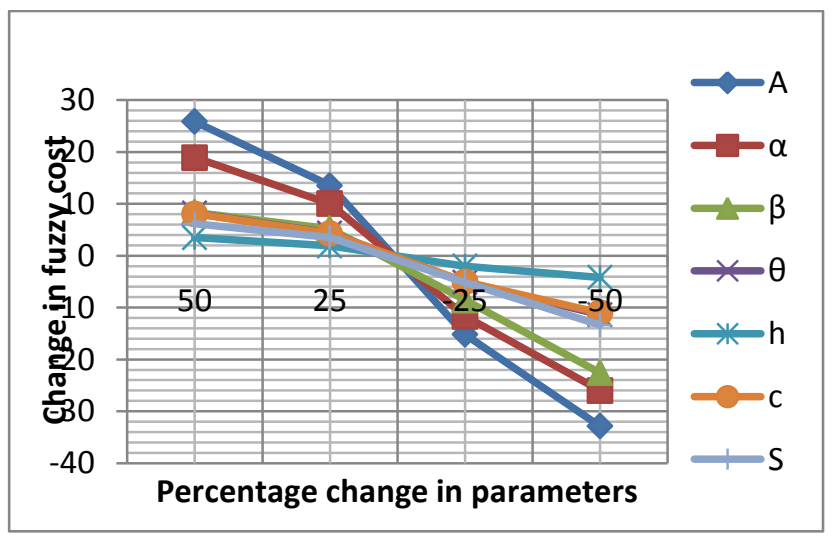

Figure 4. Behavior of fuzzy cost in signed distance method

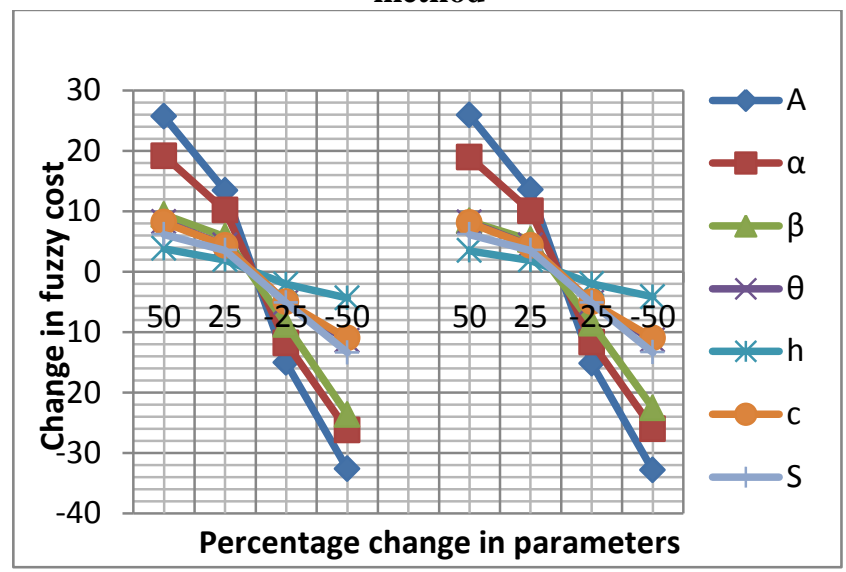

Figure 5. Comparison of fuzzy cost in both GMIM and SDM

\section{CONCLUSION}

This paper deals with optimal fuzzy replenishment model with Weibull demand rate and time dependent proportional deterioration rate. Here holding cost varies per unit of time. The demand rate, deterioration rate and various costs are taken as pentagonal fuzzy numbers. Graded mean integration method and signed distance method are used for defuzzification of total inventory cost under fuzzy sense to take proper decision. After comparison, it is concluded that graded mean integration method provides optimum inventory cost as compared to signed distance method for this model.

The general method can be applied to the inventory problem of mixture in inventory system. Numerical illustrations suggest the total fuzzy costs are significantly sensitive to the variation of length of planning horizon and system parameters. The results show that the proposed method can be an acceptable model. This model can be induced in industry dealing with production of deteriorating commodity vis-a-vis imprecision. This proposed model can be improved by introducing trade credit, non-instantaneous deterioration, discounted selling price, salvage cost, stock dependent demand and freezing technology. The results of the study give managerial insights to decision maker developing an optimal ordering decision for deterioration products. Compensation mechanism should also be included as a scope of future research to induce collaboration in the supply chain of deterioration products with limited expiration period.

\section{ACKNOWLEDGEMENT}

The second author would like to thank anonymous referees for their valuable and constructive comments and suggestions that have led to improvement on the earlier version of the paper.

\section{REFERENCES}

[1] N.P. Behera, P.K. Tripathy, "Fuzzy EOQ Model for TimeDeteriorating Items Using Penalty Cost", American Journal of Operational Research, Vol.6, No.1, pp. 1-8, 2016.

[2] S.C. Chang, J.S. Yao, H. M. Lee, "Economic reorder point for fuzzy backorder quantity", European Journal of Operational Research, Vol.109, pp.183-202, 1998.

[3] M.A. Hariga, "Optimal EOQ models for deteriorating items with time-varying demand", The Journal of the Operational Research Society, Vol.47, pp.1228-1246, 1996.

[4] C.H. Hsieh, "Optimization of fuzzy production inventory models", Information Sciences, Vol.146, pp.29-40, 2002.

[5] C.K. Jaggi, K.K. Aggarwal, S.K. Goel, "Optimal order policy for deteriorating items with inflation induced demand", International Journal of Production Economics, Vol.103, pp.707-714, 2006.

[6] C.K. Jaggi, S. Pareek, A. Sharma, Nidhi, "Fuzzy Inventory Model for Deteriorating Items with Time-varying Demand and Shortages" American Journal of Operational Research, Vol.2, No.6, pp.81-92, 2012.

[7] C. Kao, W.K. Hsu, "Lot size-reorder point inventory model with fuzzy demands", Computers and mathematics with applications, Vol.43, pp.1291-1302, 2002. 
[8] S.P. Modal, M. Mandal, "Pentagonal fuzzy number, its properties and application in fuzzy equation", Future Computing and Informatics Journal, Vol.2, pp.110-117, 2017.

[9] B.S. Mohanty, P.K. Tripathy, "Fuzzy inventory model for deteriorating items with exponentially decreasing demand under fuzzified cost and partial backlogging", International Journal of Mathematics Trends and Technology, Vol.51, No.3, pp.182-189, 2017.

[10] H. Nagar, P. Surana, "Fuzzy Inventory Model for Deteriorating Items with Fluctuating Demand and Using Inventory Parameters as Pentagonal Fuzzy Numbers", Journal of Computer and Mathematical Sciences, Vol.6, No.2, pp.55-66, 2015.

[11] A. Panda, A. Pal, "A study on pentagonal fuzzy number and its corresponding matrices", Pacific Science Review B: Humanities and Social Sciences, Vol.1, pp.1-9, 2016.

[12] K.S. Park, "Fuzzy set theoretic interpretation of economic order quantity", IIIE Transactions on Systems, Man and Cybernetics, Vol.17, pp.1082-1084, 1987.

[13] P.M. Pu, Y.M Liu, "Fuzzy Topology 1, neighborhood structure of a fuzzy point and Moore-Smith Convergence", Journal of Mathematical Analysis and Application, Vol.76, pp.571-599, 1980.

[14] N.K. Sahoo, B.S. Mohanty, P.K. Tripathy, "Fuzzy inventory model with exponential demand and time-varying deterioration", Global Journal of Pure and Applied Mathematics, Vol.12, No.3, pp.2573-2589, 2016.

[15] N.K. Sahoo, P.K. Tripathy, "An EOQ Model for Quadratic Demand Rate, Parabolic Deterioration, Time Dependent Holding Cost with Partial Backlogging", International Journal of Mathematics and its Applications, Vol.6, No.1-B, pp.325-332, 2018.

[16] N.K. Sahoo, P.K. Tripathy, "An EOQ model with three-parameter Weibull deterioration, trended demand and time varying holding cost with salvage", International Journal of Mathematics Trends and Technology, Vol.51, No.5, pp.25-29, 2017.

[17] P.K. Tripathy, W.M.Wee, P.R. Majhi, "An EOQ model with process reliability considerations", Journal of the Operational Research Society, Vol.54, pp.549-554, 2003.

[18] J.S. Yao, J. Chiang, "Inventory without backorder with fuzzy total cost and fuzzy storing cost defuzzified by centroid and signed distance", European Journal of Operation Research, Vol.148, pp.401-409, 2003.

[19] J.S. Yao, H.M. Lee, "Fuzzy inventory with or without backorder for fuzzy order quantity with trapezoidal fuzzy number", Fuzzy sets and Systems, Vol.105, pp.311-337, 1999.

[20] L.A. Zadeh, "Fuzzy Sets", Information and Control, Vol.8, pp.338-353, 1965.

\section{AUTHORS PROFILE}

P. K. Tripathy is a senior Professor of the P.G. Department of Statistics, Utkal University and Director of Centre for IT Education, Utkal University. He has 29 years of teaching experience at P.G. level and 27 years of research experience. He pursued M Sc. (Statistics), Utkal University, CPS (Population Studies), IIPS, Mumbai and Ph.D. (Statistics) from Utkal University. His field of research is Operations Research. He has published 70 research papers in international/national journals. He has produced 12 Doctorates and 6 are to be awarded.

N. K. Sahoo is working as a lecturer in department of Mathematics, Satyam Shivam Sundaram Mahavidyalaya, Dhenkanal affiliated to Utkal University, Bhubaneswar, India and doing Ph.D. in Mathematics, Utkal University. His research area is Inventory control, fuzzy logic.

\section{Note:}

This paper has been presented at National Seminar on "Statistics in Research: Trends \& Applications" organized by the P.G. Dept. of Statistics, Utkal University, Bhubaneswar in association with ORSI, Bhubaneswar chapter, on $15^{\text {th }}$ April, 2018. 